\title{
Chronic carotid glomitis and the glomus pulmonale
}

Q Khan, D Heath

\begin{abstract}
A histological study was made of the carotid bodies and glomus pulmonale in $\mathbf{5 0}$ consecutive subjects over the age of $\mathbf{5 0}$ years who came to necropsy to determine if chronic glomitis is confined to the carotid bodies or whether it also occurs in other glomera. Lymphoid aggregates and plasma cell activity were found in the glomus pulmonale just as they were in the carotid bodies. Chronic pulmonary glomitis sometimes occurred in association with chronic carotid glomitis and sometimes independently of this. The mean age of the affected subjects was 76 years in isolated pulmonary glomitis and 79 years in chronic carotid glomitis. Chronic carotid glomitis affected seven (14\%) subjects and predominated in women (six to one). In the seven cases of isolated pulmonary glomitis women predominated five to two.

Chronic glomitis seems to be a disease process which may affect at least two members of the non-chromafin paraganglionic system, but it is not clear why focal chronic inflammation affects the glomus pulmonale of one person but not of another.
\end{abstract}

We recently described a chronic carotid glomitis in which aggregates of lymphocytes up to $500 \mu \mathrm{m}$ in diameter occurred throughout the substance of the carotid bodies of elderly subjects. ${ }^{1}$ The histological appearances are reminiscent of those found in focal chronic thyroiditis suggesting that chronic carotid glomitis may be an autoimmune mediated disease. In a few cases plasma cells accumulate around glomic venules and nerve fibrils. ${ }^{2}$ This additional feature is consistent with the formation of antibodies locally in the tissue, perhaps implying that the antigen is ageing nerve tissue. We thought it would be of interest to determine if chronic glomitis was confined to the carotid bodies or whether it also occurred in other components of the non-chromaffin paraganglionic system. One readily accessible component is the so-called "glomus pulmonale" which forms a small nodule of tissue lying on the bifurcation of the pulmonary trunk.

Krahl was the first to show that a glomus exists at a constant site on this vessel. ${ }^{3} \mathrm{He}$ thought that it was the homologue of the carotid body for the derivative of the sixth branchial arch-namely, the pulmonary circulation. Becker disagreed with this view, regard- ing this nodule on the pulmonary trunk as one of the coronary glomera localised between the ascending aorta and pulmonary trunk. ${ }^{4} \mathrm{We}$ confirmed that the glomus pulmonale is supplied by the intertruncal arteries arising from the coronary arteries and aorta. ${ }^{5}$ Irrespective of the function of this glomus, however, it provides a readily accessible nodule of the nonchromaffin paraganglionic system distant from the carotid bodies. We selected it for study to investigate if it may be the site of chronic glomitis and plasma cell activity, and to determine if chronic carotid and pulmonary glomitis can coexist.

\section{Patients and methods}

The carotid bodies in 50 subjects undergoing necropsy were dissected out. Their age and sex are indicated in fig $1 ; 20$ were male. All subjects were over the age of 50 as chronic carotid glomitis is known not to occur below this age. ${ }^{1}$ Specifically the age ranged from 52-93 years.

The diagnoses of the major diseases and causes of death in these 50 subjects varied widely and are not listed here, but care was taken to exclude from the series any condition associated with chronic hypoxaemia or systemic hypertension, both of which are known to lead to characteristic histological changes of carotid body hyperplasia. ${ }^{6}$ The carotid bodies

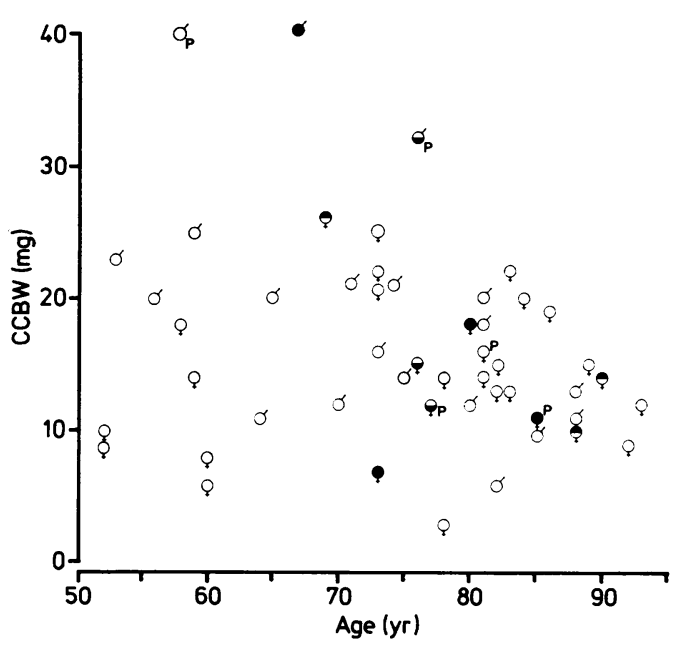

Figure 1 Graph relating the presence or absence of aggregates of lymphocytes in the carotid bodies and glomus pulmonale to the age and sex of the subjects studied and to the combined carotid body weight ( $C C B W)$.

$\mathrm{O}=$ Lymphoid aggregates in neither organ

= In both organs.

$\boldsymbol{\theta}=$ In carotid bodies alone.

$\theta=$ In glomus pulmonale alone.

Plasma cell activity indicated by $P$ 
were dissected out by the method we used previously, ${ }^{7}$ weighed, and then fixed in neutral buffered formalin, processed and embedded in paraffin wax. During the blocking out procedure the carotid body was orientated so that its largest diameter was parallel to the plane of section. Sections were cut at levels throughout the block and aggregates of lymphocytes looked for. This was a well defined histological feature, and individual cases could be designated unequivocally positive or negative. No attempt was made to grade the aggregates according to their size or numbers present in the sections. A diffuse and usually sparse scattering of lymphocytes throughout the carotid body was ignored as this is known to be a normal age change in the carotid bodies of subjects above the age of $50 .^{8} \mathrm{~A}$ search was also made for collections of plasma cells in each carotid body and its nerves.

In this same series of subjects a resection and histological examination of the glomus pulmonale was made by the technique previously reported by Edwards and Heath. ${ }^{9}$ In each case the pulmonary trunk and the proximal portions of the right and left main pulmonary arteries were resected en bloc and opened with scissors anteriorly. A block of tissue was removed just posterior and caudal to the bifurcation where there seemed to be a small plexus of nerves. This block was fixed in $10 \%$ formalin and subsequently embedded in paraffin wax. Sections $5 \mu \mathrm{m}$ in thickness were cut at 50 intervals of $50 \mu \mathrm{m}$ throughout the block so that a total length of $2.5 \mathrm{~mm}$ of pulmonary trunk was examined. Sections were stained with haematoxylin and eosin. Glomic tissue was found in the adventitia of the pulmonary trunk immediately posterior and caudal to the bifurcation. Aggregates of lymphocytes in the glomus pulmonale were looked for. As in the case of the carotid bodies this was a well defined entity and permitted unequivocal designation. No attempt was made to grade lymphocytic foci according to size or numbers present in the sections. Diffuse, sparse scattering of lymphocytes throughout the glomus pulmonale was ignored, being regarded as an age change similar to that found in the carotid bodies. A search was made for collections of plasma cells in each glomus pulmonale and its nerves.

\section{Results}

\section{QUALITATIVE HISTOLOGY}

The histological structure of the glomus pulmonale closely resembled that of the carotid body, being composed of clusters including dark, light, and "pyknotic" variants of chief (type I) cells with surrounding rims of elongated sustentacular (type II) cells. Lymphocytes were commonly seen as diffuse infiltrates of varying degrees of severity throughout the glomic substance. This was regarded as an age change. ${ }^{8}$ In some instances the lymphocytes formed aggregates in the glomic substance and in the fibrous capsule of the glomus pulmonale (fig 2) just as in cases of chronic carotid glomitis (fig 3 ). The aggregates were composed of small lymphocytes and they

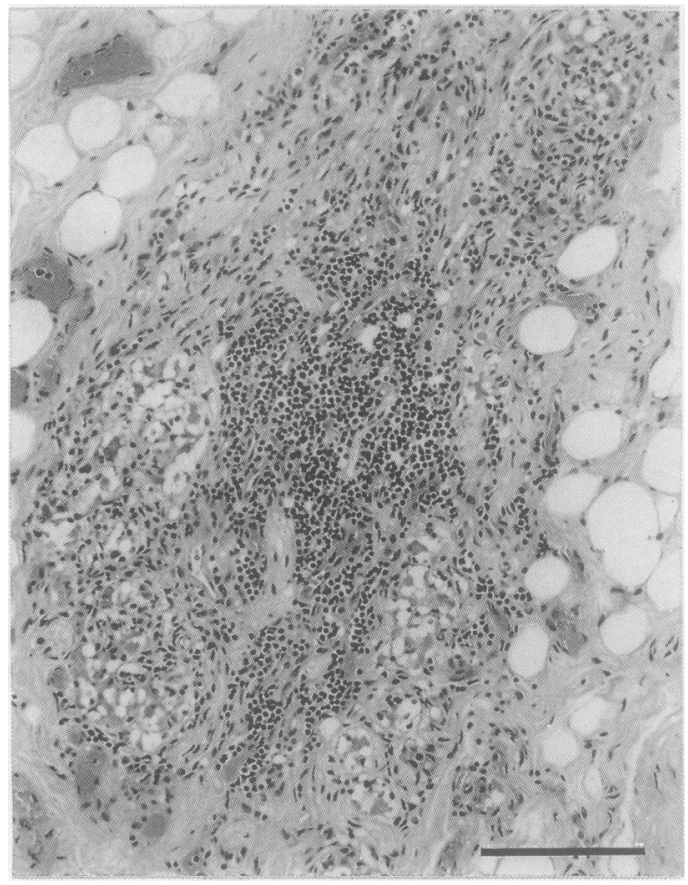

Figure 2 Glomus pulmonale from a 73 year old woman who died from metastases from a carcinoma of the gall bladder. There is a large focus of lymphocytes in the glomic parenchyma. (Haematoxylin and eosin.) Bar line $=100 \mu \mathrm{m}$

were often found around small, thin walled blood vessels. In some instances lymphocytes were found grouped around branches of nerve trunks as they entered the glomus. Diffuse and focal collections of lymphocytes were often found in the fibrofatty tissue around the glomus and often formed aggregates beneath the covering layer of mesothelium. In other instances the structure of the glomus was disturbed so that the cellular clusters had been replaced by fibrous tissue in some areas and distorted by proliferation of sustentacular cells

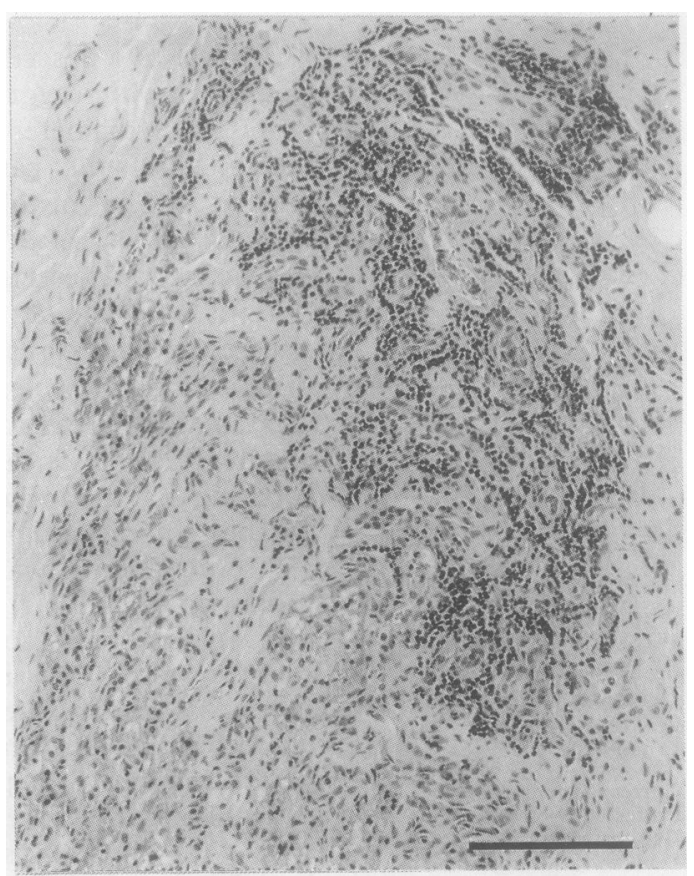

Figure 3 Carotid body from the same case. Chronic carotid glomitis is present with aggregates of lymphocytes in the glomic substance. (Haematoxylin and eosin.) Bar line $=100 \mu \mathrm{m}$. 
Figure 4 Glomus pulmonale from a 76 year (f), with loss of glomic parenchyma (p), and an infiltrate in which plasma cells were prominent. eosin.) Bar line $=100$ $\mu m$. old man showing fibrosis, (Haematoxylin and

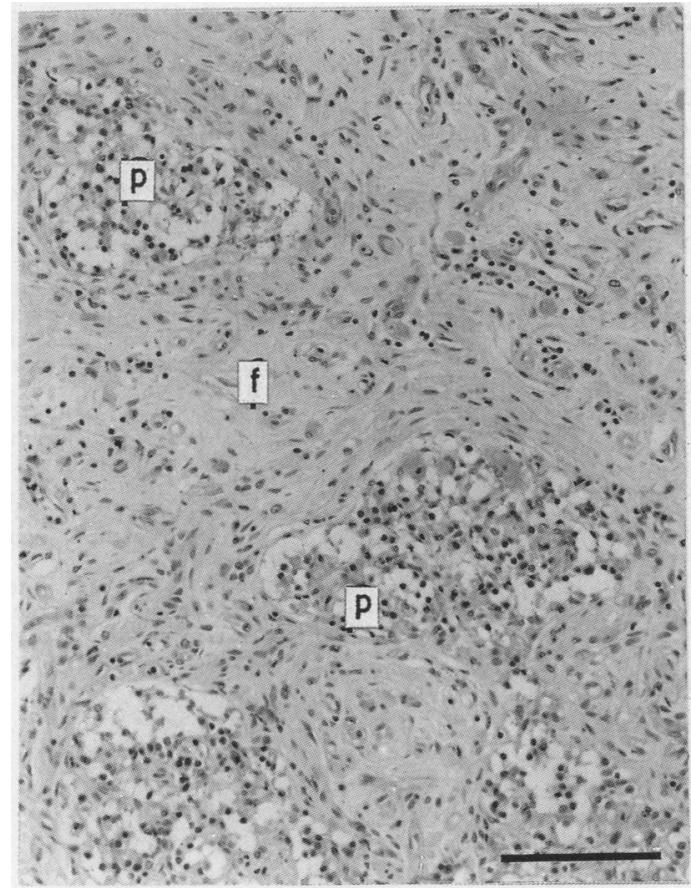

in others (fig 4). Such abnormal glomera frequently showed infiltration by plasma cells, which was also apparent in the connective tissues, particularly around nerves (fig 5).

Immunological characterisation of the lymphocytes was not carried out in this study, but had been performed in a previous investigation of a series of cases of chronic carotid glomitis. ${ }^{1}$ The small lymphocytes diffusely distributed through the stroma, with a few within the glomic tissue, were virtually all $\mathrm{T}$ cells. In one case only were there diffusely distributed B cells. Immunohistological analysis of the aggregates showed that they varied in composition, but $\mathrm{T}$ lymphocytes always comprised $50-75 \%$ of the cells present.

CORRELATION BETWEEN LYMPHOID AGGREGATES IN CAROTID BODIES AND GLOMUS PULMONALE The incidence of chronic carotid glomitis and

Figure 5 Detail of cellular infiltrate from the case illustrated in the preceding figure. As well as diffuse infiltrates of lymphocytes considerable numbers of plasma cells (arrows) are present, especially near nerve fibrils $(n)$.

(Haematoxylin and

eosin.) Bar line $=50 \mu \mathrm{m}$.

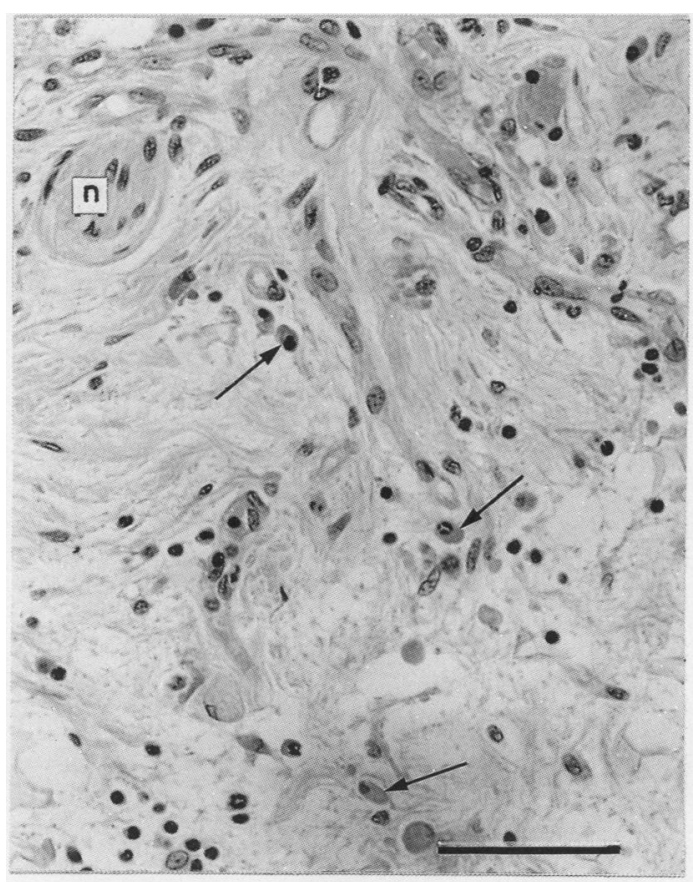

pulmonary glomitis in this series and the relation of both to age, sex, and combined carotid body weight are shown in fig 1 . This weight ranged from 3 to $40 \mathrm{mg}$. In all but two it was 32 mg or less. While we have previously suggested that the upper limit of combined carotid body weight is $30 \mathrm{mg}$, we have also pointed out that confirmation of carotid body hyperplasia depends on the presence of its characteristic histological features. ${ }^{10}$ Applying this criterion, none of the cases in this series was hyperplastic. Possibly small flecks of adipose or connective tissue, undetected at dissection, accounted for the weight exceeding $32 \mathrm{mg}$ in the two cases. Another factor to be taken into consideration is that one of the two exceptions showed chronic carotid glomitis. In a previous study we found that combined carotid body weight equalled or exceeded $30 \mathrm{mg}$ in five of six cases of chronic carotid glomitis which occurred in men. ${ }^{1}$

Chronic carotid glomitis was found in seven cases $(14 \%)$, ranging in age from 67 to 90 years, the mean age being 79 years. It was isolated in three instances $(6 \%)$ and combined with pulmonary glomitis in four instances $(8 \%)$. Isolated pulmonary glomitis occurred in three cases $(6 \%)$ in patients aged, respectively, 76, 76 , and 77 years. In the seven cases of chronic carotid glomitis women predominated six to one. In the seven cases of pulmonary glomitis women predominated five to two. Plasma cell activity was found in the glomus pulmonale in five cases (figs 4 and 5). In two of these cases lymphoid aggregates were not found in either the carotid bodies or the glomus pulmonale. In two others there was isolated pulmonary glomitis. In the fifth case focal aggregates of lymphocytes were found in the carotid bodies and the glomus pulmonale. The age range of these subjects was 58-85 years, the mean age being 75 years. Three of the five subjects showing plasma cell activity were women.

\section{Discussion}

Our study shows that lymphoid aggregates and, less commonly, infiltrates of plasma cells may be found in the glomus pulmonale just as they are in the carotid bodies where they constitute chronic carotid glomitis. ${ }^{1}$ Thus chronic glomitis seems to be a disease process which may affect at least two members of the non-chromaffin paraganglionic system and possibly more. It is not clear why focal chronic inflammation affects the glomus pulmonale of one person but not of another. Sometimes pulmonary glomitis occurs on its own and sometimes in association with chronic carotid glomitis.

Like the condition in the carotid bodies, pulmonary glomitis is a disease of the elderly. In a previous study we found the prevalence of chronic carotid glomitis to be $21 \%$ in those over the age of 50 years. $^{1}$ In the present investigation the prevalence was $14 \%$ for chronic carotid glomitis and $14 \%$ for chronic pulmonary glomitis. Such data show that lymphoid aggregates in components of the non-chromaffin paraganglionic system are already quite common in the elderly but 
increasingly occur in the aged. In the present investigation both chronic carotid and pulmonary glomitis predominated in women.

Perhaps, in view of its histological features reminiscent of chronic focal thyroiditis, chronic glomitis of the carotid bodies and glomus pulmonale is an expression of autoimmunity. As suggested for the carotid body, it may be a tissue response to ageing nerve fibrils. In the glomus pulmonale care must be taken in diagnosing glomitis, for the surrounding fibroadipose tissue, nerves, and submesothelial connective tissues may also be the site of diffuse and focal chronic inflammatory infiltrates.

This study was supported by a grant from the North West Regional Research Committee and by the Edith Walsh Award of the British Medical Association.
1 Khan Q, Heath D, Nash J, Smith P. Chronic carotid glomitis. Histopathol 1989;14:471-81.

2 Heath D, Khan $Q$, Nash J, Smith P. Carotid body disease and the physician-chronic carotid glomitis. Postgrad Med J 1989;65:353-7.

$3 \mathrm{Krahl}$ VE. The glomus pulmonale: its location and microscopic anatomy. In Ciba Foundation Symposium on pulmonary structure and function. London: Churchill pulmonary structure
Livingstone, $1962: 53$.

4 Becker AE. The glomera in the region of the heart and great vessels. A microscopic anatomical and histochemical
study. MD thesis. Laboratory of Pathological Anatomy of the University of Amsterdam, 1966.

5 Edwards C, Heath D. Microanatomy of glomic tissue of the pulmonary trunk. Thorax 1969;24:209-17. 6 Heath D, Smith P, Jago R. Hyperplasia of the carotid body.

7 Smith $P$, Jago R, Heath D. Anatomical variation and quantitative histology of the normal and enlarged carotid body. J Pathol 1982;137:287-304.

8 Hurst G, Heath D, Smith P. Histological changes associated with ageing of the human carotid body. J Pathol 1985;147: with agei $181-7$.

9 Edwards C, Heath D. Site and blood supply of the intertruncal glomera. Cardiovasc Res 1970;4:502-8.

10 Heath D, Smith P. The pathology of the carotid body and sinus. London: Edward Arnold, 1985:17. 